\title{
Separated Belyi maps
}

\author{
ZaChary ScherR AND Michael E. Zieve
}

\begin{abstract}
We construct Belyi maps having specified behavior at finitely many points. Specifically, for any curve $C$ defined over $\overline{\mathbb{Q}}$, and any disjoint finite subsets $S, T \subset C(\overline{\mathbb{Q}})$, we construct a finite morphism $\varphi: C \rightarrow \mathbb{P}^{1}$ such that $\varphi$ ramifies at each point in $S$, the branch locus of $\varphi$ is $\{0,1, \infty\}$, and $\varphi(T) \cap\{0,1, \infty\}=\emptyset$. This refines a result of Mochizuki's. We also prove an analogous result over fields of positive characteristic, and in addition we analyze how many different Belyi maps $\varphi$ are required to imply the above conclusion for a single $C$ and $S$ and all sets $T \subset C(\overline{\mathbb{Q}}) \backslash S$ of prescribed cardinality.
\end{abstract}

\section{Introduction}

Let $C$ be a (smooth, projective, geometrically irreducible) algebraic curve defined over $\mathbb{C}$. Belyi [2] gave an unexpected necessary and sufficient condition for $C$ to be isomorphic to a curve defined over $\overline{\mathbb{Q}}$ : namely, that there should exist a finite morphism $\varphi: C \rightarrow \mathbb{P}^{1}$ which has exactly three branch points. We refer to such a map $\varphi$ as a Belyi map, and write $\operatorname{Br}(\varphi)$ for its branch locus. Belyi maps have had important consequences to topics ranging from Galois theory [7] to physics [1]; see for instance [3, 5, 6, 12-15] for various other consequences of Belyi maps. In some applications, one needs Belyi maps $\varphi$ which satisfy additional properties. One prominent example is Mochizuki's inter-universal Teichmüller theory [10], which relies on his earlier results on Belyi maps [11].

We are interested in studying the amount of flexibility there is in the choice of a Belyi map $\varphi$ on a prescribed curve $C$. One way to measure this is through the preimage $\varphi^{-1}(\operatorname{Br}(\varphi))$. We will show in Proposition 3.4 that, for any curve $C$ of genus at least 2 , only finitely many subsets of $C(\mathbb{C})$ of any prescribed cardinality can occur as $\varphi^{-1}(\operatorname{Br}(\varphi))$ for a Belyi map $\varphi$ on $C$. These distinguished finite subsets of $C(\mathbb{C})$ are the focus of this paper.

We now state our first main result. Throughout this paper, we view all curves as coming equipped with a fixed embedding into projective space, 
so that if a curve $C$ is defined over a field $K$ then we can speak of the coordinatewise action of the absolute Galois group of $K$ on points of $C(\bar{K})$.

Theorem 1.1. Let $K$ be a number field, let $C$ be a curve over $K$, and let $S$ and $T$ be finite subsets of $C(\bar{K})$ such that $S$ is disjoint from the set of $\operatorname{Gal}(\bar{K} / K)$-conjugates of elements of $T$. Then there exists a finite morphism $\varphi: C \rightarrow \mathbb{P}^{1}$ defined over $K$ such that

- $\operatorname{Br}(\varphi)=\{0,1, \infty\}$,

- $\varphi$ is ramified at every point in $S$,

- $\varphi(T) \cap\{0,1, \infty\}=\emptyset$.

In this result, the set $\{0,1, \infty\}$ could be replaced by any prescribed threeelement subset of $K$, since there are linear fractional transformations over $K$ which map any such subset to $\{0,1, \infty\}$. In [11, Theorem 2.5], Mochizuki proved a similar result in which the condition that $\varphi$ be ramified on $S$ is replaced by the weaker condition that $\varphi(S)=\{0,1, \infty\}$. It is natural to attempt to deduce our result from Mochizuki's by replacing Mochizuki's map $\varphi_{0}$ with the composition $\psi \circ \varphi_{0}$ where $\psi: \mathbb{P}^{1} \rightarrow \mathbb{P}^{1}$ is ramified at each point in $\{0,1, \infty\}$ and also $\operatorname{Br}(\psi)=\{0,1, \infty\}$ and $\psi^{-1}(\{0,1, \infty\})=\{0,1, \infty\}$; however, in fact no such map $\psi$ exists, so one must use a different argument. Our proof of Theorem 1.1 is quite different from Mochizuki's proof of [11, Theorem 2.5], and uses ideas from [4], although both our proof and Mochizuki's follow the general outline of all known proofs of Belyi's theorem.

Positive characteristic analogues of Belyi maps seem to have been considered for the first time by Katz [8, Lemma 16] in the context of the Langlands correspondence for function fields. In positive characteristic, every curve admits a finite morphism to $\mathbb{P}^{1}$ having just one branch point. We prove the following analogue of Theorem 1.1 in this setting.

Theorem 1.2. Let $K$ be a perfect field of characteristic $p>0$, let $C$ be a curve over $K$, and let $S, T$ be finite subsets of $C(\bar{K})$ such that $S$ is disjoint from the set of $\operatorname{Gal}(\bar{K} / K)$-conjugates of elements of $T$. Then there exists a finite morphism $\varphi: C \rightarrow \mathbb{P}^{1}$ defined over $K$ such that

- $\operatorname{Br}(\varphi)=\{\infty\}$,

- $\varphi$ is ramified at every point in $S$,

- $\infty \notin \varphi(T)$. 
Now we fix a set $S$ and let $T$ vary over all subsets of $C(\overline{\mathbb{Q}}) \backslash S$ of prescribed cardinality $n$. In Theorem 1.1, we produced a Belyi map corresponding to any such $T$; our next result shows that in fact only $n+1$ Belyi maps are needed to account for all $T$.

Theorem 1.3. Let $n \geq 1$ be an integer, and let $C$ be a curve defined over a number field $K$. For any finite $\operatorname{Gal}(\bar{K} / K)$-stable subset $S$ of $C(\bar{K})$, there exist finite morphisms

$$
\varphi_{1}, \ldots, \varphi_{n+1}: C \rightarrow \mathbb{P}^{1}
$$

defined over $K$ such that

- $\operatorname{Br}\left(\varphi_{i}\right)=\{0,1, \infty\}$ for $1 \leq i \leq n+1$,

- each $\varphi_{i}$ is ramified at every point in $S$,

- every $n$-element subset $T \subset C(\bar{K}) \backslash S$ satisfies $\varphi_{i}(T) \cap\{0,1, \infty\}=\emptyset$ for at least one $i \in\{1,2, \ldots, n+1\}$ (where $i$ may depend on $T$ ).

This refines a result of Mochizuki's [11, Corrollary 3.1], which shows the existence of a finite set of Belyi maps satisfying the above properties, except with our condition on the ramification of $\varphi_{i}$ replaced by the weaker condition $\varphi_{i}(S)=\{0,1, \infty\}$. Mochizuki's proof uses a compactness argument which does not provide control on the number of maps required; we use a different argument which enables us to determine the minimum number of maps possible. In Theorem 5.1, we give an analogous result in positive characteristic. We note that these results have a topological interpretation. For any curve $C$ defined over $\overline{\mathbb{Q}}$, we say that a Belyi open subset of $C(\overline{\mathbb{Q}})$ is any set of the form $C(\overline{\mathbb{Q}}) \backslash \varphi^{-1}(\operatorname{Br}(\varphi))$ where $\varphi: C \rightarrow \mathbb{P}^{1}$ is a Belyi map. Theorem 1.1 implies that, for any disjoint finite $S, T \subset C(\overline{\mathbb{Q}})$, there is a Belyi open set which contains $T$ but is disjoint from $S$. Theorem 1.3 implies that, for any finite $S \subset C(\overline{\mathbb{Q}})$, there are $n+1$ Belyi open sets $U_{1}, \ldots, U_{n+1}$ such that any $n$-element subset $T$ of $C(\overline{\mathbb{Q}}) \backslash S$ satisfies $T \subset U_{i} \subseteq C(\overline{\mathbb{Q}}) \backslash S$ for some $i$.

Belyi constructed a Belyi map $\varphi$ on any curve $C$ over $\overline{\mathbb{Q}}$ by writing $\varphi=\varphi_{3} \circ \varphi_{2} \circ \varphi_{1}$, where $\varphi_{1}: C \rightarrow \mathbb{P}^{1}$ is any finite morphism defined over $\overline{\mathbb{Q}}$, $\varphi_{2}: \mathbb{P}^{1} \rightarrow \mathbb{P}^{1}$ satisfies $B:=\operatorname{Br}\left(\varphi_{2}\right) \cup \varphi_{2}\left(\operatorname{Br}\left(\varphi_{1}\right)\right) \subset \mathbb{Q}$, and $\varphi_{3}: \mathbb{P}^{1} \rightarrow \mathbb{P}^{1}$ satisfies $\operatorname{Br}\left(\varphi_{3}\right) \cup \varphi_{3}(B)=\{0,1, \infty\}$. His argument shows that there exist Belyi maps $\varphi$ for which $\varphi^{-1}(\operatorname{Br}(\varphi))$ contains any prescribed subset of $C(\overline{\mathbb{Q}})$. Both Mochizuki's proof and our proof have a similar structure to Belyi's proof, in that $\varphi$ is constructed as the composition of three maps. The difference is 
that we require some points to be outside the branch loci of the maps $\varphi_{i}$, as in the following diagram (where we assume $T \neq \emptyset$ ):

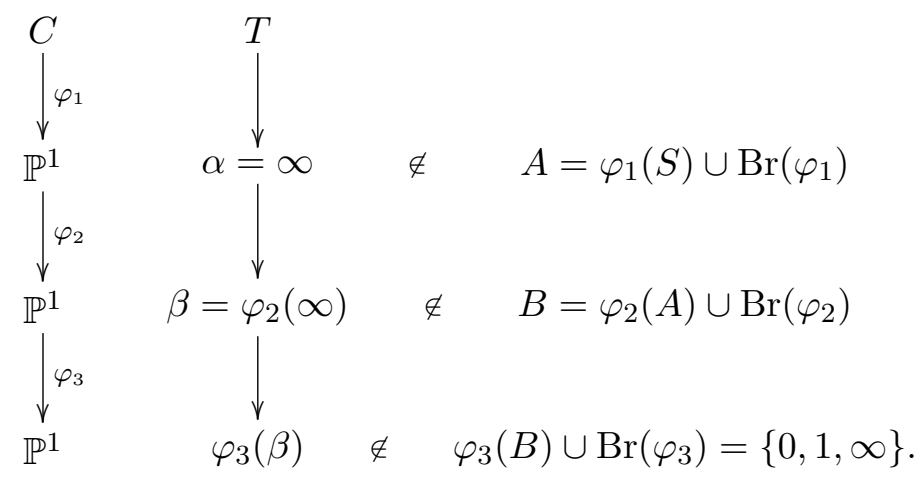

We will construct the map $\varphi_{1}$ in the next section, treating the case of positive characteristic at the same time as the case of characteristic zero. The maps $\varphi_{2}$ and $\varphi_{3}$ are constructed for fields of characteristic zero in the section after that, and then we construct them over fields of positive characteristic. Then in the last section we treat collections of Belyi maps.

\section{Reduction to $\mathbb{P}^{1}$}

Our goal is to show that every curve $C$ defined over a suitable field admits a Belyi map $C \rightarrow \mathbb{P}^{1}$ with prescribed behavior at finitely many points. In this section, we show that it suffices to do this when $C=\mathbb{P}^{1}$.

Proposition 2.1. Let $C$ be a curve defined over a perfect field $K$. If $S$ and $T$ are disjoint finite $\operatorname{Gal}(\bar{K} / K)$-stable subsets of $C(\bar{K})$ then there exists a finite morphism

$$
\varphi: C \rightarrow \mathbb{P}^{1}
$$

defined over $K$ such that $\varphi(T) \subseteq\{\infty\}$ and $\infty \notin \varphi(S) \cup \operatorname{Br}(\varphi)$.

Before proving this we recall some terminology. A divisor on $C$ (over $\bar{K}$ ) is said to be defined over $K$ if it is fixed by $\operatorname{Gal}(\bar{K} / K)$. For any divisor $D$ on $C$ which is defined over $K$, the associated Riemann-Roch space is the $K$-vector space

$$
\mathcal{L}(D):=\{f \in K(C):(f) \geq-D\} \cup\{0\}
$$

and the dimension of this vector space is denoted $\ell(D)$. 
Proof. We may (and will) assume that $T$ is nonempty, since the result for empty $T$ follows from the result for nonempty $T$. Let $O_{1}, O_{2}, \ldots, O_{n}$ be the $\operatorname{Gal}(\bar{K} / K)$-orbits of points in $T$, and for each $i$ let $D_{i}$ be the divisor

$$
D_{i}=\sum_{P \in O_{i}} P
$$

Note that $D_{i}$ is defined over $K$. Let $g$ be the genus of $C$, and let $R$ be a finite $\operatorname{Gal}(\bar{K} / K)$-stable subset of $C(\bar{K})$ such that $|R| \geq 2 g-1$ and $R \cap(S \cup T)=$ $\emptyset$. Let $D$ be the divisor

$$
D=\sum_{P \in R} P
$$

so that $D$ is defined over $K$ and $\operatorname{deg}(D)=|R| \geq 2 g-1$. By the following lemma, there is an element $f \in \mathcal{L}\left(D+\sum_{i=1}^{n} D_{i}\right)$ which is not in $\mathcal{L}(D+$ $\left.\sum_{i \neq j} D_{i}\right)$ for any $j$. Then $f \in K(C)$ has simple poles at all the points of $T$, at most simple poles at the points in $R$, and no other poles. Because $R$ is disjoint from $S$, we see that $f$ extends to a morphism $\varphi$ satisfying the requirements of the proposition.

Lemma 2.2. Let $C$ be a curve of genus $g$ defined over a perfect field $K$. Let $T$ be a non-empty finite $\operatorname{Gal}(\bar{K} / K)$-stable subset of $C(\bar{K})$. Let $O_{1}, \ldots, O_{n}$ be the $\operatorname{Gal}(\bar{K} / K)$-orbits in $T$, and let $D_{i}$ be the divisor $D_{i}=\sum_{P \in O_{i}} P$. If $D$ is a divisor defined over $K$ of degree at least $2 g-1$, then

$$
\mathcal{L}\left(D+\sum_{i=1}^{n} D_{i}\right) \supsetneqq \bigcup_{j=1}^{n} \mathcal{L}\left(D+\sum_{i \neq j} D_{i}\right) .
$$

Proof. For each $j \in\{1,2, \ldots, n\}$, we have

$$
\operatorname{deg}\left(D+\sum_{i=1}^{n} D_{i}\right)>\operatorname{deg}\left(D+\sum_{i \neq j} D_{i}\right) \geq 2 g-1
$$

so the Riemann-Roch theorem implies that

$$
\ell\left(D+\sum_{i=1}^{n} D_{i}\right)=\operatorname{deg}(D)+|T|+1-g>\ell\left(D+\sum_{i \neq j} D_{i}\right)
$$


Since a vector space over an infinite field cannot be written as the union of finitely many proper subspaces, it follows that if $K$ is infinite then

$$
\mathcal{L}\left(D+\sum_{i=1}^{n} D_{i}\right) \supsetneqq \bigcup_{j=1}^{n} \mathcal{L}\left(D+\sum_{i \neq j} D_{i}\right) .
$$

Henceforth assume that $K$ is finite, and write

$$
\begin{aligned}
m_{i} & :=\operatorname{deg}\left(D_{i}\right)=\left|O_{i}\right|, \\
m & :=|T|, \\
q & :=|K|, \\
r & :=\operatorname{deg}(D)+1-g .
\end{aligned}
$$

The Riemann-Roch theorem implies that $\ell\left(D+\sum_{i=1}^{n} D_{i}\right)=r+m$, and thus

$$
\left|\mathcal{L}\left(D+\sum_{i=1}^{n} D_{i}\right)\right|=q^{r+m}
$$

For distinct $j_{1}, j_{2}, \ldots, j_{k} \in\{1,2, \ldots, n\}$, we have

$$
\bigcap_{t=1}^{k} \mathcal{L}\left(D+\sum_{i \neq j_{t}} D_{i}\right)=\mathcal{L}\left(D+\sum_{i \neq j_{1}, \ldots, j_{k}} D_{i}\right) .
$$

Riemann-Roch implies that

$$
\begin{aligned}
& \ell\left(D+\sum_{i \neq j_{1}, \ldots, j_{k}} D_{i}\right)=r+\sum_{i \neq j_{1}, \ldots, j_{t}} m_{i} \\
& =r+m-m_{j_{1}}-\cdots-m_{j_{k}},
\end{aligned}
$$

so that

$$
\left|\mathcal{L}\left(D+\sum_{i \neq j_{1}, \ldots, j_{k}} D_{i}\right)\right|=q^{r+m-m_{j_{1}}-\cdots-m_{j_{k}}}
$$

It follows by inclusion-exclusion that

$$
\left|\bigcup_{j=1}^{n} \mathcal{L}\left(D+\sum_{i \neq j} D_{i}\right)\right|=\sum_{k=1}^{n}(-1)^{k+1} \sum_{1 \leq j_{1}<\ldots<j_{k} \leq n} q^{r+m-\sum_{t=1}^{k} m_{j_{t}}} .
$$


This cardinality equals

$$
q^{r+m}\left(1-\prod_{i=1}^{n}\left(1-\frac{1}{q^{m_{j_{i}}}}\right)\right)
$$

which is strictly smaller than $q^{r+m}$. Thus our union of subspaces is a proper subset of $\mathcal{L}\left(D+\sum_{i=1}^{n} D_{i}\right)$, as desired.

\section{Characteristic 0}

In this section, we prove that for any finite subset $A \subset \mathbb{P}^{1}(\overline{\mathbb{Q}})$, and any element $\alpha \in \mathbb{P}^{1}(\overline{\mathbb{Q}}) \backslash A$, there is a Belyi map $\varphi: \mathbb{P}^{1} \rightarrow \mathbb{P}^{1}$ with $\varphi(\alpha) \notin \operatorname{Br}(\varphi)=$ $\{0,1, \infty\}$ such that all points in $A$ ramify under $\varphi$. We do this in two steps. The first step produces a map $\varphi_{2}$ which allows us to reduce to the case that $A$ and $\alpha$ are in $\mathbb{P}^{1}(\mathbb{Q})$. The second step produces a map $\varphi_{3}$ for which $\varphi=\varphi_{3} \circ \varphi_{2}$ has the required properties.

To proceed, fix an embedding $\overline{\mathbb{Q}} \hookrightarrow \mathbb{C}$, and let $|\cdot|: \overline{\mathbb{Q}} \rightarrow \mathbb{R}$ be the induced absolute value. The following lemma enables us to control the absolute values of $\alpha$ and of the elements of $A$.

Lemma 3.1. Let $A$ be a finite subset of $\mathbb{P}^{1}(\overline{\mathbb{Q}})$, and pick any $\alpha \in \mathbb{P}^{1}(\mathbb{Q}) \backslash$ $A$. Then for any real number $c>1$, there exists a linear fractional transformation $\psi \in \mathbb{Q}(x)$ such that $\psi(\alpha) \in \mathbb{Q}$ with $\max _{\beta \in A}\{|\psi(\beta)|\}<1$ and $|\psi(\alpha)|>c$.

Proof. Upon replacing $A$ and $\alpha$ by their images under a suitably chosen linear fractional transformation in $\mathbb{Q}(x)$, we may assume that $\alpha=0$ and $\infty \notin A$. Because $0=\alpha \notin A$, we can choose $r \in \mathbb{Q}$ with $0<r<\min _{\beta \in A} \frac{|\beta|}{c+1}$. It follows that every $\beta \in A$ satisfies

$$
r c<|\beta|-r
$$

so the triangle inequality yields

$$
r c<|\beta|-r \leq|\beta-r|
$$

Choose a rational number $s$ such that $r c<s<\min _{\beta \in A}|\beta-r|$. Now the linear fractional transformation

$$
\psi(x):=\frac{s}{x-r}
$$


satisfies

$$
|\psi(0)|=\frac{s}{r}>c
$$

and, for $\beta$ in $A$,

$$
|\psi(\beta)|=\frac{s}{|\beta-r|}<1 .
$$

Since $s$ and $r$ are rational, $\psi$ satisfies the conditions of the lemma.

Our next result enables us to reduce from the case of a finite subset of $\mathbb{P}^{1}(\overline{\mathbb{Q}})$ to a finite subset of $\mathbb{P}^{1}(\mathbb{Q})$.

Proposition 3.2. Let $A$ be a finite subset of $\mathbb{P}^{1}(\overline{\mathbb{Q}})$, and let $\alpha \in \mathbb{P}^{1}(\mathbb{Q}) \backslash A$. There exists a rational function $f \in \mathbb{Q}(x)$ such that

$$
f(A), \operatorname{Br}(f), f(\alpha) \subseteq \mathbb{P}^{1}(\mathbb{Q}) \quad \text { and } \quad f(\alpha) \notin f(A) \cup \operatorname{Br}(f) .
$$

Proof. For any $\alpha \in \mathbb{P}^{1}(\mathbb{Q})$ and any finite subsets $A_{0}, A_{1}$ of $\mathbb{P}^{1}(\overline{\mathbb{Q}}) \backslash\{\alpha\}$ with $A_{0} \subset A_{1}$, the conclusion of the Proposition holds for $A=A_{0}$ if it holds for $A=A_{1}$. Therefore we may assume without loss that $A$ is nonempty. Moreover, the hypothesis $\alpha \in \mathbb{P}^{1}(\mathbb{Q}) \backslash A$ implies that $\alpha$ is not in the image of $A$ under $\operatorname{Gal}(\overline{\mathbb{Q}} / \mathbb{Q})$, so (since this image is finite) we may assume that $A$ is preserved by $\operatorname{Gal}(\overline{\mathbb{Q}} / \mathbb{Q})$. Let $c>1$ be a constant whose value will be chosen later. Then Lemma 3.1 produces a degree-one rational function $\psi(x) \in \mathbb{Q}(x)$ for which $\psi(\alpha) \in \mathbb{Q},|\psi(\alpha)|>c$, and $\max _{\beta \in \bar{A}}\{|\psi(\beta)|\}<1$. It follows that $\hat{A}:=\psi(A)$ is a subset of $\overline{\mathbb{Q}}$ which does not contain the rational number $\hat{\alpha}:=\psi(\alpha)$, and moreover (since $\psi(x) \in \mathbb{Q}(x)$ ) that $\hat{A}$ is preserved by $\operatorname{Gal}(\overline{\mathbb{Q}} / \mathbb{Q})$. Note that $|\hat{A}| \leq|A|$ is bounded by a constant which does not depend on the choice of $c$. If $\hat{f} \in \mathbb{Q}(x)$ satisfies $\hat{f}(\hat{A}), \operatorname{Br}(\hat{f}), \hat{f}(\hat{\alpha}) \subseteq \mathbb{P}^{1}(\mathbb{Q})$ and $\hat{f}(\hat{\alpha}) \notin \hat{f}(\hat{A}) \cup \operatorname{Br}(\hat{f})$, then $\hat{f} \circ \psi$ satisfies all the properties required of $f$. Thus, there is no loss in replacing $A$ and $\alpha$ by $\hat{A}$ and $\hat{\alpha}$, so we may assume that $\alpha \in \mathbb{Q}$, that $A$ is a $\operatorname{Gal}(\overline{\mathbb{Q}} / \mathbb{Q})$-stable subset of $\overline{\mathbb{Q}}$, and in addition that $\max _{\beta \in A}\{|\beta|\}<1$ and $|\alpha|>c$.

Writing $n:=|A|$ (which is bounded by a constant which does not depend on the choice of $c$ ), we will define polynomials $f_{0}, f_{1}, \ldots, f_{n-1} \in \mathbb{Q}[x]$ such that

(1) $\operatorname{deg}\left(f_{i}\right)=n-i$ for $0 \leq i \leq n-1$,

(2) $f_{i}\left(\operatorname{Br}\left(f_{i-1}\right)\right)=\{0, \infty\}$ for $1 \leq i \leq n-1$,

(3) $f_{0}(A)=\{0\}$,

(4) the leading coefficient of $f_{i}$ is an integer, 
(5) all coefficients of $f_{i}$ have absolute value bounded by an absolute constant (independent of the choice of $c$ ) for $0 \leq i \leq n-1$.

Suppose for the moment that such $f_{i}$ have been constructed. By properties (1) and (5) there is a constant $N$, independent of $c$, such that for each $i$ all coefficients of $f_{n-1} \circ f_{n-2} \circ \cdots \circ f_{i}$ have absolute value at most $N$. We now show that if $c$ is sufficiently large then $f:=f_{n-1} \circ f_{n-2} \circ \cdots \circ f_{1} \circ f_{0}$ satisfies the conclusion of the Proposition. For $\beta \in A$ we have $f_{0}(\beta)=0$ and thus $f(\beta)=f_{n-1} \circ \cdots \circ f_{1}(0)$ is a rational number of absolute value at most $N$. Next, any $\delta \in \operatorname{Br}(f) \backslash\{\infty\}$ has the form $\delta=f_{n-1} \circ f_{n-2} \circ \cdots \circ f_{i}(\gamma)$ where $0 \leq i \leq n-2$ and $f_{i}^{\prime}(\gamma)=0$. Property (2) implies that $f_{i+1}\left(f_{i}(\gamma)\right)=0$, so that $\delta$ equals $f_{n-1} \circ f_{n-2} \circ \cdots \circ f_{i+2}(0)$ and hence is a rational number of absolute value at most $N$. Since the leading coefficient of $f(x)$ has absolute value at least 1 , and all other coefficients have absolute value at most $N$, it follows that if $c$ is sufficiently large then $|f(\alpha)|>N$. But we have shown that $f(A) \cup \operatorname{Br}(f)$ consists of rational numbers of absolute value at most $N$, so we must have $f(\alpha) \notin f(A) \cup \operatorname{Br}(f)$. Finally, since $f(x) \in$ $\mathbb{Q}[x]$ and $\alpha \in \mathbb{Q}$, we see that $f(\alpha) \in \mathbb{Q}$, which implies the conclusion of the proposition.

To finish the proof, we must construct polynomials $f_{i} \in \mathbb{Q}[x]$ satisfying the stated properties. Define

$$
f_{0}(x):=\prod_{\beta \in A}(x-\beta) .
$$

Since $A$ is $\operatorname{Gal}(\overline{\mathbb{Q}} / \mathbb{Q})$-stable, $f_{0}$ must be in $\mathbb{Q}[x]$. Moreover, $f_{0}$ is monic of degree $n$ and $f_{0}(A)=\{0\}$. Since $|\beta|<1$ for all $\beta \in A$, and every coefficient of $f_{0}$ is the sum of several products of distinct $\beta$ 's (or the negative of such a sum), it follows that every coefficient of $f_{0}$ has absolute value less than $2^{n}$. Therefore $f_{0}$ satisfies the required properties. Inductively, suppose we have defined $f_{i-1}$ for some $i$ with $1 \leq i \leq n-1$, and that $f_{i-1}$ satisfies the required properties. Then define

$$
f_{i}(x):=\operatorname{Res}_{y}\left(f_{i-1}^{\prime}(y), f_{i-1}(y)-x\right)
$$

where $\operatorname{Res}_{y}(*, *)$ is the resultant with respect to the variable $y$. Since the resultant is the determinant of the Sylvester matrix, it follows that $f_{i}$ has rational coefficients and that all its coefficients have absolute value at most some function of $n$ (independent of $c$ ). Let $a x^{d}$ be the leading term of $f_{i-1}(x)$, where we know that $d=n-i+1$. Let $r_{1}, r_{2}, \ldots, r_{d-1}$ be the roots of $f_{i-1}^{\prime}$, 
counted with multiplicity. Then we have

$$
f_{i}(x)=(a d)^{d} \prod_{j=1}^{d-1}\left(f_{i-1}\left(r_{j}\right)-x\right) .
$$

It follows that the leading term of $f_{i}$ is $(-x)^{d-1}(a d)^{d}$, and that the roots of $f_{i}$ are precisely the finite branch points of $f_{i-1}$. Thus $f_{i}$ satisfies Properties (1)(5), so by induction these properties hold for $f_{0}, f_{1}, \ldots, f_{n-1}$, which implies the result.

We now construct the desired Belyi map in case the curve is $\mathbb{P}^{1}$ and all distinguished points are in $\mathbb{P}^{1}(\mathbb{Q})$.

Proposition 3.3. Let $B$ be a finite subset of $\mathbb{P}^{1}(\mathbb{Q})$, and pick any $\beta \in$ $\mathbb{P}^{1}(\mathbb{Q}) \backslash B$. Then there exists a rational function $f \in \mathbb{Q}(x)$ such that

- $f$ is ramified at every point in $B$,

- $\operatorname{Br}(f)=\{0,1, \infty\}$,

- $f(\beta) \notin\{0,1, \infty\}$.

Proof. Upon replacing $B$ and $\beta$ by their images under $\frac{1}{x-a}$ for any prescribed $a \in \mathbb{Q} \backslash(B \cup\{\beta\})$, we may assume that $B \subset \mathbb{Q}$ and $\beta \in \mathbb{Q}$. Upon replacing $B$ and $\beta$ by their images under $a x$ for a suitable $a \in \mathbb{Z} \backslash\{0\}$, we may assume that $B \subset \mathbb{Z}$ and $\beta \in \mathbb{Z}$. By adjoining to $B$ a sufficiently large finite subset of $\mathbb{Z} \backslash(B \cup\{\beta\})$, we may assume that $|B| \geq 2$. Let $p$ be a prime which does not divide $\gamma-\beta$ for any $\gamma \in B$, and put $\delta:=\beta+p$. Let $B^{\prime}:=B \cup\{\delta\}$, and write the elements of $B^{\prime}$ as

$$
B^{\prime}=\left\{b_{1}, b_{2}, \ldots, b_{m}\right\}
$$

with $b_{m}=\delta$.

We now construct the desired rational function $f(x)$. Let

$$
c:=\prod_{1 \leq i<j \leq m}\left(b_{i}-b_{j}\right),
$$

and note that $c \neq 0$. Partial fraction decomposition ensures that there are unique $n_{1}, \ldots, n_{m} \in \mathbb{Q}$ such that

$$
\sum_{i=1}^{m} \frac{n_{i}}{x-b_{i}}=\frac{c}{\prod_{j=1}^{m}\left(x-b_{j}\right)},
$$


namely

$$
n_{i}:=\frac{c}{\prod_{\substack{1 \leq j \leq m \\ j \neq i}}\left(b_{i}-b_{j}\right)} .
$$

Our choice of $c$ ensures that the $n_{i}$ are nonzero integers. Finally, we define

$$
f(x):=\prod_{i=1}^{m}\left(x-b_{i}\right)^{2 n_{i}} .
$$

It remains to check that $f(x)$ has the required properties. Since each $n_{i}$ is nonzero, the elements of $B^{\prime}$ are critical points of $f$. Every finite critical point of $f$ must be either a zero or a pole of

$$
\frac{f^{\prime}(x)}{f(x)}=\sum_{i=1}^{m} \frac{2 n_{i}}{x-b_{i}} .
$$

Our construction of the $n_{i}$ 's ensures that the right side equals

$$
\frac{2 c}{\prod_{j=1}^{m}\left(x-b_{j}\right)},
$$

so $f$ cannot have any critical points outside of $B^{\prime} \cup\{\infty\}$. Since $f\left(B^{\prime}\right) \subseteq$ $\{0, \infty\}$ and $f(\infty) \in\{0,1, \infty\}$, it follows that $\operatorname{Br}(f) \subseteq\{0,1, \infty\}$. Moreover, since $f$ ramifies at each element of $B^{\prime}$ and we know that $\left|B^{\prime}\right| \geq 3$, RiemannHurwitz implies that $f$ must have at least three branch points, whence $\operatorname{Br}(f)=\{0,1, \infty\}$. Finally, we show that $f(\beta) \notin\{0,1, \infty\}$. Since $\beta \notin B^{\prime}$, we know a priori that $f(\beta) \notin\{0, \infty\}$. Our choice of the prime $p$ ensures that $p \nmid\left(\beta-b_{i}\right)$ for $i<m$, yet $p \mid\left(\beta-b_{m}\right)$. Thus the $p$-adic valuation of

$$
f(\beta)=\prod_{i=1}^{m}\left(\beta-b_{i}\right)^{2 n_{i}}
$$

is nonzero, so that $f(\beta) \neq 1$.

With all the ingredients in place, we can now prove Theorem 1.1.

Proof of Theorem 1.1. By adjoining to $S$ the set of $\operatorname{Gal}(\bar{K} / K)$-conjugates of elements of $S$, we may assume that $S$ is preserved by $\operatorname{Gal}(\bar{K} / K)$. Likewise we may assume that $T$ is preserved by $\operatorname{Gal}(\bar{K} / K)$. By adjoining to $T$ a finite $\operatorname{Gal}(\overline{\mathbb{Q}} / K)$-stable set of points in $C(\overline{\mathbb{Q}}) \backslash S$ if necessary, we may assume that $T$ is nonempty. Let $\varphi_{1}: C \rightarrow \mathbb{P}^{1}$ be the map produced by Proposition 2.1 
for this choice of $S$ and $T$. Then $\varphi_{1}$ is defined over $K$, the set $A:=\varphi_{1}(S) \cup$ $\operatorname{Br}\left(\varphi_{1}\right)$ does not contain $\alpha:=\infty$, and $\varphi_{1}(T)=\{\infty\}$. Let $\varphi_{2}: \mathbb{P}^{1} \rightarrow \mathbb{P}^{1}$ be the map produced by Proposition 3.2 for $A$ and $\alpha$. Then $\varphi_{2}$ is defined over $\mathbb{Q}$, both $\beta:=\varphi_{2}(\alpha)$ and $B:=\varphi_{2}(A) \cup \operatorname{Br}\left(\varphi_{2}\right)$ are contained in $\mathbb{P}^{1}(\mathbb{Q})$, and $\beta \notin B$. Lastly, let $\varphi_{3}: \mathbb{P}^{1} \rightarrow \mathbb{P}^{1}$ be the map produced by Proposition 3.3 for $B$ and $\beta$. Then $\varphi_{3}$ is defined over $\mathbb{Q}$, every point in $B$ ramifies under $\varphi_{3}$, and $\varphi_{3}(\beta) \notin\{0,1, \infty\}=\operatorname{Br}\left(\varphi_{3}\right)$. Pictorially, we have

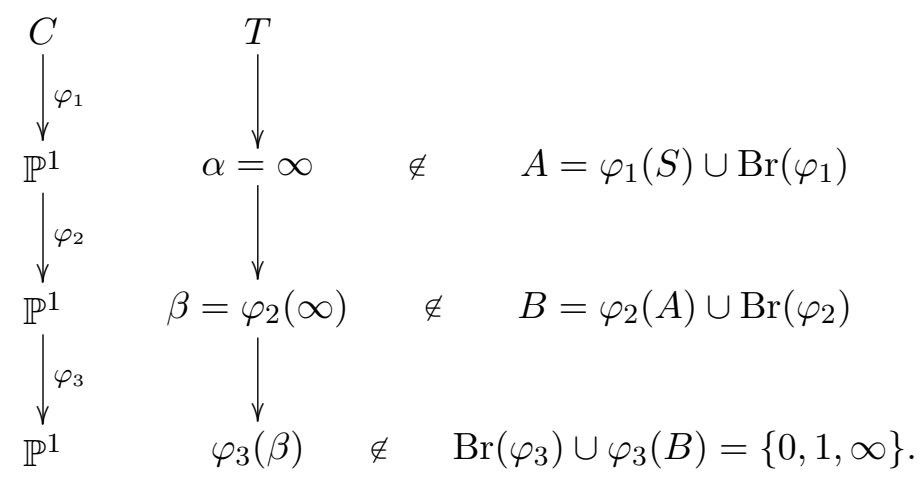

Thus $\varphi:=\varphi_{3} \circ \varphi_{2} \circ \varphi_{1}$ is a finite morphism $C \rightarrow \mathbb{P}^{1}$ defined over $K$, and $\varphi(T)=\varphi_{3}(\beta) \notin\{0,1, \infty\}$. Since $\varphi_{2}\left(\varphi_{1}(S)\right) \subseteq B$ and every point of $B$ ramifies under $\varphi_{3}$, it follows that every point of $S$ ramifies under $\varphi$. Finally, $\operatorname{Br}(\varphi)$ is the union of the three sets $\operatorname{Br}\left(\varphi_{3}\right), \varphi_{3}\left(\operatorname{Br}\left(\varphi_{2}\right)\right)$, and $\varphi_{3}\left(\varphi_{2}\left(\operatorname{Br}\left(\varphi_{1}\right)\right)\right)$, and hence equals $\{0,1, \infty\}$.

We conclude this section by showing that, if $C$ is a complex curve of genus at least 2 , then only finitely many subsets of $C(\mathbb{C})$ of any prescribed cardinality can occur as $\varphi^{-1}(\operatorname{Br}(\varphi))$ where $\varphi: C \rightarrow \mathbb{P}^{1}$ is a Belyi map. In fact, we will show at the same time that only finitely many curves $C$ of any prescribed genus admit a Belyi map $\varphi: C \rightarrow \mathbb{P}^{1}$ for which $\varphi^{-1}(\operatorname{Br}(\varphi))$ has prescribed cardinality.

Proposition 3.4. Fix integers $n, g \geq 1$. There are only finitely many isomorphism classes of complex curves $C$ of genus $g$ for which there exists a Belyi map $\varphi: C \rightarrow \mathbb{P}^{1}$ such that $\left|\varphi^{-1}(\operatorname{Br}(\varphi))\right|=n$. Moreover, if $g \geq 2$ and $C$ is a curve of genus $g$, then there are only finitely many n-element subsets of $C(\mathbb{C})$ which occur as $\varphi^{-1}(\operatorname{Br}(\varphi))$ for a Belyi map $\varphi: C \rightarrow \mathbb{P}^{1}$.

Proof. Let $\varphi: C \rightarrow \mathbb{P}^{1}$ be a degree- $d$ Belyi map on a genus- $g$ curve $C$, and let $B:=\varphi^{-1}(\operatorname{Br}(\varphi))$. For any $P \in B$, write $e(P)$ for the ramification index 
of $P$ under $\varphi$. The Riemann-Hurwitz formula implies that

$$
2 g-2=-2 d+\sum_{P \in B}(e(P)-1)=-2 d+3 d-|B|
$$

so that $d=2 g-2+|B|$. Thus, $d$ is determined by $g$ and $n:=|B|$.

For any Belyi map $\varphi: C \rightarrow \mathbb{P}^{1}$, there is a linear fractional transformation $\mu \in \mathbb{C}(x)$ for which $\mu(\operatorname{Br}(\varphi))=\{0,1, \infty\}$. Thus $\bar{\varphi}:=\mu \circ \varphi$ is a Belyi map with branch locus $\{0,1, \infty\}$, and $\varphi^{-1}(\operatorname{Br}(\varphi))=\bar{\varphi}^{-1}(\{0,1, \infty\})$. So there is no loss in restricting to Belyi maps with branch locus $\{0,1, \infty\}$. A classical result (see for instance, [9, Prop. 3.1]) implies that, for any fixed $d$, there are only finitely many isomorphism classes of pairs $(C, \varphi)$ where $C$ is a complex curve and $\varphi: C \rightarrow \mathbb{P}^{1}$ is a degree- $d$ Belyi map with branch locus $\{0,1, \infty\}$. Thus, for fixed $n$ and $g$, there are only finitely many isomorphism classes of corresponding curves $C$. Since any curve of genus at least 2 has only finitely many automorphisms, it follows that for fixed $n, g$ with $g \geq 2$ and a fixed genus- $g$ curve $C$ there are only finitely many $n$-element sets of the form $\varphi^{-1}(\{0,1, \infty\})$, where $\varphi: C \rightarrow \mathbb{P}^{1}$ is a Belyi map with branch locus $\{0,1, \infty\}$.

\section{Characteristic $p$}

In this section, we prove Theorem 1.2. The key tool is the following result:

Proposition 4.1. Let $K$ be a perfect field of characteristic $p>0$, and let $B \subset \mathbb{P}^{1}(\bar{K})$ be a finite set such that $0 \notin B$. Then there exists $f \in K(x)$ such that

- $f$ is ramified at every point in $B$

- $\operatorname{Br}(f)=\{\infty\}$

- $f(0) \neq \infty$.

Proof. Since the result in case $B=\emptyset$ follows from the result in case $B=\{1\}$, we may assume that $B$ is nonempty. Let $\bar{B} \subset \mathbb{P}^{1}(\bar{K})$ be the set of $\operatorname{Gal}(\bar{K} / K)$ conjugates of elements in $B \backslash\{\infty\}$, and let $V \subset \bar{K}$ be the $\mathbb{F}_{p}$-span of $\bar{B}$. Then $V$ is a finite set which is preserved by $\operatorname{Gal}(\bar{K} / K)$, and its minimal polynomial

$$
T(x):=\prod_{\alpha \in V}(x-\alpha)
$$


has the form

$$
T(x)=\sum_{i=0}^{n} a_{i} x^{p^{i}},
$$

where $a_{i} \in K$ and $a_{0} \neq 0$. Let

$$
S(x):=\frac{T(x)^{p}}{x^{p}}=\sum_{i=0}^{n} a_{i}^{p} x^{p\left(p^{i}-1\right)}
$$

and

$$
g(x):=x^{p}+\frac{1}{T(x)+S(x)} .
$$

The rational function $g(x)$ is well defined since $S(0)=a_{0}^{p} \neq 0$, while $T(0)=$ 0 . Plainly $g(\infty)=\infty$. Since $0 \notin B$ by hypothesis, for $\beta$ in $B \backslash\{\infty\}$ we have

$$
T(\beta)+S(\beta)=T(\beta)+\frac{T(\beta)^{p}}{\beta^{p}}=0,
$$

so that $g(\beta)=\infty$. Thus $g(B) \subseteq\{\infty\}$, and furthermore

$$
g(0)=\frac{1}{T(0)+S(0)}=\frac{1}{a_{0}^{p}} \neq \infty .
$$

Since $T^{\prime}(x)=a_{0}$ and $S^{\prime}(x)=0$, we compute

$$
g^{\prime}(x)=\frac{-a_{0}}{(T(x)+S(x))^{2}}
$$

so that $g^{\prime}(x)$ has no roots in $\bar{K}$ since $a_{0} \neq 0$. Therefore $g(x)$ has no finite critical points which are not poles, and since $g(\infty)=\infty$, it follows that $\operatorname{Br}(g) \subseteq\{\infty\}$. The polynomial $x^{p}+x \in K[x]$ has $\infty$ as its unique critical point and its unique branch point. Thus $f:=g^{p}+g$ satisfies the requirements of the proposition.

Proof of Theorem 1.2. By adjoining to $S$ the set of $\operatorname{Gal}(\bar{K} / K)$-conjugates of elements of $S$, we may assume that $S$ is preserved by $\operatorname{Gal}(\bar{K} / K)$. Likewise we may assume that $T$ is preserved by $\operatorname{Gal}(\bar{K} / K)$. By adjoining to $T$ a finite $\operatorname{Gal}(\bar{K} / K)$-stable set of points in $C(\bar{K}) \backslash S$ if necessary, we may assume that $T$ is nonempty. Let $\varphi_{1}: C \rightarrow \mathbb{P}^{1}$ be the map constructed in Proposition 2.1 for this choice of $S$ and $T$. Then $\varphi_{1}$ is defined over $K$, the set $A:=\varphi_{1}(S) \cup$ $\operatorname{Br}\left(\varphi_{1}\right)$ does not contain $\alpha:=\infty$, and $\varphi_{1}(T)=\{\infty\}$. Let $\varphi_{2}: \mathbb{P}^{1} \rightarrow \mathbb{P}^{1}$ be the map $x \mapsto 1 / x$, and put $B:=\varphi_{2}(A)$, so that $B$ does not contain $\varphi_{2}(\alpha)=0$. Let $\varphi_{3}: \mathbb{P}^{1} \rightarrow \mathbb{P}^{1}$ be the map constructed in Proposition 4.1 for this choice 
of $B$. Then $\varphi_{3}$ is defined over $K$, ramifies at every point in $B$, and satisfies $\varphi_{3}(0) \neq \infty$ and $\operatorname{Br}\left(\varphi_{3}\right)=\{\infty\}$. This yields the diagram

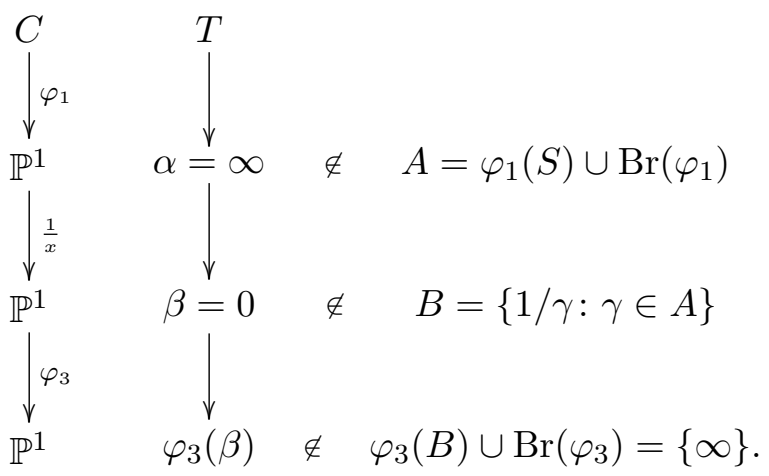

Then $\varphi:=\varphi_{3} \circ \varphi_{2} \circ \varphi_{1}$ is a finite morphism $C \rightarrow \mathbb{P}^{1}$ defined over $K$, and $\varphi(T)=\varphi_{3}(\beta) \neq \infty$. Since $\varphi_{3}$ ramifies at every point in $B$, and $B$ contains $\varphi_{2}\left(\varphi_{1}(S)\right)$, we see that $\varphi$ ramifies at every point in $S$. Finally, since $\varphi_{2}$ is unramified, $\operatorname{Br}(\varphi)$ is the union of $\operatorname{Br}\left(\varphi_{3}\right)$ and $\varphi_{3}\left(\varphi_{2}\left(\operatorname{Br}\left(\varphi_{1}\right)\right)\right)$, and hence equals $\{\infty\}$.

\section{Collections of Belyi maps}

Finally, we consider collections of Belyi maps, and prove Theorem 1.3.

Proof of Theorem 1.3. Let $T_{0}=\emptyset$, and use the following inductive procedure to define finite subsets $T_{i}$ of $C(\overline{\mathbb{Q}}) \backslash S$ and morphisms $\varphi_{i}: C \rightarrow \mathbb{P}^{1}$ for each $i=1,2, \ldots, n+1$. For any $i \in\{1,2, \ldots, n+1\}$ such that $T_{i-1}$ has been defined, let $\varphi_{i}: C \rightarrow \mathbb{P}^{1}$ be the morphism produced by Theorem 1.1 for the sets $S$ and $T:=T_{i-1}$. Then $\varphi_{i}$ is a finite morphism defined over $K$ such that

- $\operatorname{Br}\left(\varphi_{i}\right)=\{0,1, \infty\}$,

- $\varphi_{i}$ is ramified at every point in $S$,

- $\varphi_{i}\left(T_{i-1}\right) \cap\{0,1, \infty\}=\emptyset$.

Define

$$
T_{i}:=T_{i-1} \cup \varphi_{i}^{-1}(\{0,1, \infty\}) \backslash S,
$$

and note that $T_{i}$ is a finite subset of $C(\overline{\mathbb{Q}}) \backslash S$. This procedure yields morphisms $\varphi_{1}, \ldots, \varphi_{n+1}: C \rightarrow \mathbb{P}^{1}$. For each $i \in\{1,2, \ldots, n+1\}$, note that

$$
\varphi_{i}^{-1}(\{0,1, \infty\})=S \cup\left(T_{i} \backslash T_{i-1}\right)
$$


Since $T_{1} \subseteq T_{2} \subseteq \cdots \subseteq T_{n+1}$, the sets

$$
T_{1} \backslash T_{0}, T_{2} \backslash T_{1}, \ldots, T_{n+1} \backslash T_{n}
$$

are pairwise disjoint. Thus any $n$-element subset $T$ of $C(\overline{\mathbb{Q}})$ must be disjoint from at least one set $T_{i} \backslash T_{i-1}, \quad$ so if $T \cap S=\emptyset$ then $\varphi_{i}(T) \cap$ $\{0,1, \infty\}=\emptyset$.

The following positive characteristic analogue of Theorem 1.3 can be shown by a similar argument.

Theorem 5.1. Let $n$ be a positive integer, and let $C$ be a curve defined over a perfect field $K$ of characteristic $p>0$. For any finite $\operatorname{Gal}(\bar{K} / K)$ stable subset $S$ of $C(\bar{K})$, there exist finite morphisms

$$
\varphi_{1}, \ldots, \varphi_{n+1}: C \rightarrow \mathbb{P}^{1}
$$

defined over $K$ such that

- $\operatorname{Br}\left(\varphi_{i}\right)=\{\infty\}$ for $1 \leq i \leq n+1$,

- each $\varphi_{i}$ is ramified at every point in $S$,

- every $n$-element subset $T \subset C(\bar{K}) \backslash S$ satisfies $\varphi_{i}(T) \cap\{\infty\}=\emptyset$ for at least one $i \in\{1,2, \ldots, n+1\}$ (where $i$ may depend on $T$ ).

We conclude with a remark about potential improvements of the number $n+1$ of maps in Theorem 1.3.

Remark. Let $C$ be a curve over $\overline{\mathbb{Q}}$, and let $S$ be a finite subset of $C(\overline{\mathbb{Q}})$. If $S$ has the form $\varphi^{-1}(\operatorname{Br}(\varphi))$ for some Belyi map $\varphi: C \rightarrow \mathbb{P}^{1}$, then we may replace the $n+1$ maps $\varphi_{1}, \ldots, \varphi_{n+1}$ in the conclusion of Theorem 1.3 with the single map $\varphi$. If $S$ does not have this form, then the $n+1$ Belyi maps in the conclusion of Theorem 1.3 cannot be replaced by a smaller set of maps. For, if $\varphi_{1}, \ldots, \varphi_{n}: C \rightarrow \mathbb{P}^{1}$ are Belyi maps with $\operatorname{Br}\left(\varphi_{i}\right)=\{0,1, \infty\}$ and $S \varsubsetneqq \varphi_{i}^{-1}(\{0,1, \infty\})$, then pick any $t_{i} \in \varphi_{i}^{-1}(\{0,1, \infty\}) \backslash S$, and note that

$$
T:=\left\{t_{1}, \ldots, t_{n}\right\}
$$

satisfies $|T| \leq n$ and

$$
\varphi_{i}(T) \cap\{0,1, \infty\} \neq \emptyset
$$

for every $i$. 


\section{Acknowledgments}

The authors thank the referee for reading this paper extremely carefully and providing several helpful comments. The authors thank the NSF for support under grants EMSW21-RTG:0943832 and DMS-1162181.

\section{References}

[1] S.K. Ashok, F. Cachazo and E. Dell'Aquila, Children's drawings from Seiberg-Witten curves, Commun. Number Theory Phys. 1(2) (2007), 237-305.

[2] G.V. Belyi, On Galois extensions of a maximal cyclotomic field, Math. USSR Izv. 14 (1980), 247-256.

[3] I.I. Bouw, S. Wewers and L. Zapponi, Deformation data, Belyi maps, and the local lifting problem, Trans. Amer. Math. Soc. 361(12) (2009), 6645-6659.

[4] E.H. Brooks, J.H. Rosen, Z. Scherr, B.L. Weiss and M.E. Zieve, $A$ classification of certain Belyi maps, Preprint.

[5] N.D. Elkies, Shimura curves for level-3 subgroups of the $(2,3,7)$ triangle group, and some other examples, in 'Algorithmic number theory', Lecture Notes in Computer Science, 4076, 302-316, Springer, Berlin, 2006.

[6] W. Goldring, Unifying themes suggested by Belyi's theorem, in 'Number theory, analysis and geometry', 181-214, Springer, New York, 2012.

[7] A. Grothendieck, Esquisse d'un programme, in 'Geometric Galois actions, 1', London Mathematical Society Lecture Note Series, 242, 5-48, Cambridge University Press, Cambridge, 1997. With an English translation on pp. 243-283.

[8] N.M. Katz, Travaux de Laumon, Astérisque (1988), no. 161-162, Exp. No. 691, 4, 105-132 (1989). Séminaire Bourbaki, Vol. 1987/88.

[9] B. Köck, Belyi's theorem revisited, Beiträge Algebra Geom. 45(1) (2004), 253-265.

[10] S. Mochizuki, Inter-universal Teichmüller theory IV: log-volume computations and set-theoretic foundations, Preprint. http://www. kurims.kyoto-u.ac.jp/ motizuki/papers-english.html 
[11] —, Noncritical Belyi maps, Math. J. Okayama University 46 (2004), 105-113.

[12] K. Nakanishi, Lamé operators with projective octahedral and icosahedral monodromies, Rend. Sem. Mat. Univ. Padova 114 (2005), 109-129 (2006).

[13] L. Schneps (ed.), The Grothendieck theory of dessins d'enfants, London Mathematical Society Lecture Note Series, 200, Cambridge University Press, Cambridge, 1994, ISBN 0-521-47821-9. Papers from the Conf. on Dessins d'Enfant held in Luminy, April 19-24, 1993.

[14] L. Schneps and P. Lochak (eds.), Geometric Galois actions', 1, Vol. 242 of London Mathematical Society Lecture Note Series, Cambridge University Press, Cambridge, 1997, ISBN 0-521-59642-4. Around Grothendieck's "Esquisse d'un programme".

[15] - Geometric Galois actions', 2, Vol. 243 of London Mathematical Society Lecture Note Series, Cambridge University Press, Cambridge (1997), ISBN 0-521-59641-6. The inverse Galois problem, moduli spaces and mapping class groups.

Department of Mathematics

University of Pennsylvania

209 South 33RD StreEt

PhilADELPHIA, PA 19104-6395

USA

E-mail address: zscherr@math.upenn.edu

URL: http://www. math. upenn.edu/ zscherr/

Department of Mathematics

UNIVERSITY OF MiCHIGAN

530 Church Street

ANN ARBOR, MI 48109-1043

USA

Mathematical Sciences Center

TSINGHua University

BEIJING 100084

China

E-mail address: zieve@umich.edu

URL : http://www.math.lsa.umich.edu/ zieve/

Received November 14, 2013 